\title{
Solvent Free and Catalyst Free Synthesis of Octahydroquinazolinone via Grindstone Chemistry: A Total Green Approach
}

\author{
TANAY PRAMANIK ${ }^{\star 1}$, KOUNSAR MAJEED ${ }^{1}$ and RICHA GUPTA ${ }^{1}$ \\ 'Department of Chemistry, Faculty of Technology and Science, Lovely Professional University, \\ Phagwara, Punjab-144411, India. \\ ${ }^{*}$ Corresponding author E-mail: tanay.pramanik@gmail.com \\ http://dx.doi.org/10.13005/ojc/330459
}

(Received: June 02, 2017; Accepted: June 30, 2017)

\begin{abstract}
Octahydroquinazolinone is a heterocyclic compound of immense importance in the field of medicinal and pharmaceutical chemistry. Although a large number of methods are reported in literature for synthesis of Octahydroquinazolinone via one pot multi component cyclo condensation reaction of dimedone, thio urea and aromatic aldehydes, grindstone synthesis of this heterocyclic compound is not reported till date. Hereby a green method is reported for the very first time for grindstone synthesis of Octahydroquinazolinone in a completely solvent free and catalyst free condition. A series of derivatives of this heterocyclic compounds were synthesized using various derivatives of aromatic aldehydes as reactants. After completion of reaction the crude product was re-crystallized from hot ethanol to obtain pure compound which were further characterized by melting point, IR and NMR spectroscopy.
\end{abstract}

Keyworks- Octahydroquinazolinone, green synthesis, grindstone chemistry.

\section{INTRODUCTION}

For last few decades Octa hydro quinazolinone which is an $\mathrm{N}$-containing heterocyclic compound has attracted much attention from the researchers. Thanks to its diverse therapeutic ${ }^{1}$, antimicrobial $^{2,3}$ analgesic $^{4}$ and antiinflammatory ${ }^{5}$ activities.

The most convenient and easy method for synthesis of octahydroquinazolinone is one pot multi component cyclo condensation Biginelli reaction employing equimolar quantity of dimedone, thio urea and aromatic aldehydes as reactants. Several methods which have been developed by the researchers involve the usage of non eco friendly catalyst ${ }^{6}$ and toxic solvents ${ }^{7}$ for synthesis of this heterocyclic molecule. As part of our ongoing process for development of environmentally benign synthetic protocol for biginelli reaction, various eco friendly and novel methodology such as "solvent free and catalyst free Biginelli", "Biginelli reaction in fruit juice medium"9-10, "microwave ${ }^{11}$ and light ${ }^{12}$ assisted biginelli reaction 11 " has already been developed and 
reported by our research group but green synthesis of octahydroquinazolinone has never been attempted by our research group till date.

Multi component reaction via grindstone methodology is well known and well documented in literature. Grindstone technique is considered to be environmentally benign method as this method completely avoids the usage of any solvent. Literature reported $^{13,14}$ Biginelli reactions via grindstone chemistry have made use of toxic chemicals like p-TSA as catalyst and our extensive literature search has revealed that synthesis of octahydroquinazolinone via grindstone methodology in completely solvent free and catalyst free condition is not reported in literature till date. So the need of developing a new and green methodology for grindstone synthesis of this heterocycles in completely solvent and catalyst free condition is still there.

Thus hereby we are reporting a solvent and catalyst free green synthesis of octahydroquinazolinone via grindstone chemistry for the very first time ever.

\section{EXPERIMENTAL}

The starting materials were obtained from the suppliers and were used for our reaction without doing any more purification.

\section{General method for synthesis}

As indicated in scheme-1, a mixture of 0.02 mole aldehyde, 0.02 mole of dimidone and 0.02 mole of thiourea was grinded together thoroughly in an appropriate size of mortar pestle for specific time period. All the three reactants were initially solid but after grinding for some time the texture of the reaction mixture changes to semi solid. The progress of the reaction was monitored by checking TLC after every 10 minutes of grinding process. After completing of the reaction as indicated by TLC, the crude product obtained was re crystallized from hot ethanol to obtain pure products as white solid powder.

After re crystallization, the pure products were characterized by using NMR, IR, and melting point. The observed data were identical to those of reported one.

\section{Characterization}

(4-chlorophenyl)-7,7-dimethyl-2-thioxo$1,2,3,4,7,8$-hexahydroquinazolin-5(6H)-one [Compound 4C]<smiles>CC1(C)CC(=O)C2=C(C1)NC(=S)NC2c1ccc(Cl)cc1</smiles>

Melting point- $178{ }^{\circ} \mathrm{C}$

IR (neat)

2960, 1585.54, 1093, 1587, 1458, 669.32

$\mathrm{cm}^{-1}$

${ }^{1} \mathrm{H}-\mathrm{NMR}$ (400 MHz, DMSO-d ${ }^{6}$ )

$\delta 1.00(\mathrm{t}, 6 \mathrm{H}), 2.48(\mathrm{~d}, 2 \mathrm{H}), 4.2(\mathrm{~s}, 1 \mathrm{H})$, 7.15(d, 2H), $7.23(\mathrm{NH}) 7.25(\mathrm{~d}, 2 \mathrm{H})$.

4-(4-bromophenyl)-7,7-dimethyl-2-thioxo$1,2,3,4,7,8$-hexahydroquinazolin- $5(6 \mathrm{H})$-one [Compound 4B]<smiles>CC1(C)CC(=O)C2=C(C1)NC(=S)NC2c1ccc(Br)cc1</smiles><smiles>CC1(C)CC(=O)CC(=O)C1I</smiles>

Scheme 1: Grindstone synthesis of Octahydroquinazolinone 
Melting point- $186^{\circ} \mathrm{C}$

IR (neat)

$585,1159,1508,1589,2953 \mathrm{~cm}^{-1}$

${ }^{1} \mathrm{H}-\mathrm{NMR}$ (400 MHz, DMSO-d $)$

1.00(t, 6H), 2.2(d, 2H), 2.48(d, 2H), 4.21

(s, $1 \mathrm{H}), 6.99(\mathrm{~d}, 4 \mathrm{H}), 7.35(\mathrm{~d}, 4 \mathrm{H}), 7.38(\mathrm{NH})$.

7,7-dimethyl-4-(4-nitrophenyl)-2-thioxo1,2,3,4,7,8-hexahydroquinazolin-5(6H)-one [Compound 4N]<smiles>CC1(C)CC(=O)C2=C(C1)NC(=S)NC2c1ccc([N+](=O)[O-])cc1</smiles>

Melting point- $213^{\circ} \mathrm{C}$

IR (neat)

$1144,1477,1590,2875,2960 \mathrm{~cm}^{-1}$

${ }^{1} \mathrm{H}-\mathrm{NMR}$ (400 MHz, DMSO-d ${ }^{6}$ )

$1.00(\mathrm{t}, 6 \mathrm{H}), 2.31(\mathrm{~d}, 2 \mathrm{H}), 2.48(\mathrm{~d}, 2 \mathrm{H}), 4.3$

$(\mathrm{s}, 1 \mathrm{H}), 7.31(\mathrm{NH}) 7.33(\mathrm{~d}, 2 \mathrm{H}), 8.07(\mathrm{~d}, 2 \mathrm{H})$

4-(2-Nitrophenyl)-2thioxo-1,2, 3, 4, 7, 8hexahydroquinazolin-5(6H)-one[Compound 2N]

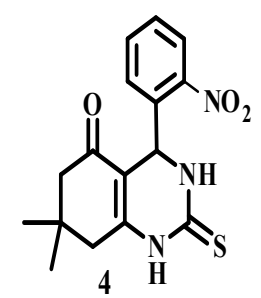

Melting point- $192^{\circ} \mathrm{C}$

IR (neat)

$3255,3115,2954,1720,1523,1617$ $1144.79 \mathrm{~cm}^{-1}$

${ }^{1} \mathrm{H}-\mathrm{NMR}$ (400 MHz, DMSO-d ${ }^{6}$ )

0.89(t, 6H), 2.0(d, 2H), 2.35(d, 2H), 4.52(s, 1H), 7.43(d, 2H), 7.76(d, 2H), $7.19(\mathrm{NH})$.

\section{RESULT AND DISCUSSION}

4 different derivates of benzaldehydes were used as reactants along with thio urea and dimedone, for synthesis of 4 different derivatives of quinazolinone via grindstone methodology. It was observed that the nature and type of the benzaldehyde used as reactant, has a great influence on the rate of the reaction.

At first the grindstone synthesis using chloro benzaldehyde as reactant, was performed in presence as well as in absence of catalyst. This is an acid catalyst reaction and clove is acidic in nature so catalytic amount of clove was used as catalyst for performing our grindstone Biginelli reaction where the cloves were grinded along with equimolar mixture of the three reactants inside the mortar. From the literature reported ${ }^{8}$ mechanism [Scheme-2] of the Biginelli reaction it is clearly evident that the presence of acid can make this reaction feasible. Considering the fact that our concerned reaction of this report follows the same mechanism as that of scheme-2, this reaction is performed in presence of mild acidic substance.

Clove has already been used in literature ${ }^{15}$ as catalyst for Biginelli reaction in water medium but clove has never been used as catalyst for any solvent free grindstone Biginelli reaction. So this is the first of its kind study where clove is being used as catalyst for a completely solvent free grindstone Biginelli reaction.

After completion of reaction (as indicated by TLC), the crude products were re crystallized from hot ethanol to obtain pure products. The same reaction was then repeated without using any clove or any other catalyst. The results observed in presence and absence of catalyst are summarized in Table-1

As observed from table-1, the reaction takes almost same time (1.5 hour) to go for completion in presence as well as in absence of catalyst. So the presence of clove as catalyst does not make any significant difference on the rate of reaction.

As the catalyst is making no significant difference on the reaction rate, the rest of the series 
Table 1: Grindstone synthesis in presence and absence of clove catalyst

\begin{tabular}{llllc}
\hline Aldehyde & Catalyst & Time & $\%$ yield & Melting point \\
\hline $\begin{array}{l}\text { Chloro } \\
\text { benzyldehyde }\end{array}$ & Clove & 1 and half hour & $85 \%$ & $174-176^{\circ} \mathrm{C}$ \\
$\begin{array}{l}\text { Chloro } \\
\text { benzyldehyde }\end{array}$ & -1 and half hour & $83 \%$ & $174-176^{\circ} \mathrm{C}$ \\
\hline
\end{tabular}<smiles>[R]C(=O)CC([R])=O</smiles>

Scheme 2: General mechanism of Biginelli reaction $^{8}$

of grindstone reactions with 4 different aromatic aldehydes were performed without using any catalyst.

The results obtained for the grindstone reactions (in absence of catalyst) with four different benzaldehydes are summarized in Table-2.

As seen from table-2, the rate of reaction is greatly influenced by the electronic nature of the benzaldehyde. Reaction with 2-nitro benzaldehyde takes 50 mins time to go for completion under grindstone condition whereas the same reaction with 4-chloro benzaldehyde takes 1.5 hours time to go for completion under identical reaction condition.
Table 2: Grindstone synthesis with different benzaldehydes in absence of catalysts and solvents

\begin{tabular}{llll}
\hline Ar group & Time & $\%$ yield & $\begin{array}{l}\text { Melting } \\
\text { point }\end{array}$ \\
\hline 4-chloro phenyl & 1.5 hours & $80 \%$ & 177 \\
4-bromo phenyl & 70 mins & $85 \%$ & 185 \\
4-nitro phenyl & 60 mins & $79 \%$ & 213 \\
2-nitro phenyl & 50 mins & $83 \%$ & 190 \\
\hline
\end{tabular}

Nitro group shows mesomeric effect which is much stronger than inductive effect shown by halogens. Thus it's the mesomeric (-M) effect of nitro group which makes the reaction of nitro benzaldehyde faster than the reaction of halo benzaldehyde. In fact with increase in electron withdrawing strength of the substituent the electrophilicity of the carbonyl centre increases that in turn increase the rate of reaction.

Although no external source of heat energy was supplied to this reaction mixture, the heat energy generated while doing the grinding of reactants using mortar and pestle supplies the necessary energy of activation for this reaction.

\section{CONCLUSION}

Herein a simple, eco friendly, cost effective, efficient and green methodology is developed for the very first time by our group for the synthesis of octahydroquinazolinone via solvent free and catalyst free grindstone method. Till date this is the first of its kind study where a grindstone multi component condensation reaction is being performed in a completely solvent free and catalyst free condition.

Although the usage of clove as catalyst for Biginelli reaction is well documented in literature ${ }^{15}$, our present study has successfully proved that the 
reaction in absence of catalyst is as good as that of in presence of catalyst.

Our current work has opened a new door of opportunity for the researchers to carry out many such multi component reactions in near future via grindstone method by completely avoiding solvent and catalyst.
So hopefully those days are not far away when we will be able to save our environment by completely avoiding the toxic effects of solvents and catalysts while doing organic reactions.

\section{ACKNOWLEDGEMENT}

Immense financial and technical support from Department of Chemistry, Lovely Professional University is gratefully acknowledged.

\section{REFRENCES}

1. Yakhontov, L.N.; Liberman, S.S.; Zhikhareva, G.P.; Kuz'mina, K.K.

Khim.-Farm. Zh., 1977, 11, 14.

2. Kung, P.P.; Casper, M.D.; Cook, K.L.; Lingardo, L.W.; Risen, L.M.;

Vickers, T.A. J. Med. Chem., 1999, 42 , 4705.

3. Alagarsamy, V.G.; Muruganathan, R.V. Biol. Pharm.

Bull. 2003, 26, 1711.

4. Nigam, R.; Swarup, S.; Saxena, V.K. Indian Drugs, 1990, 27, 38.

5. Marakun, M. Y. Japan Kokai, 1917, 77, 51.

6. Dallinger, D.; Kappe. O. Nature Protocols, 2007, 2, 317.

7. Hassani, Z.; Islami, M. R.; Kalantari, M. Bioorganic \& Medicinal Chemistry Letters, 2006, 16, 4479.

8. Pramanik, T.; Wani, T. A.; Singh, A. Orient $J$
Chem., 2013, 29, 1209

9. Pramanik, T.; Pathan, A. H. Res J Pharm Biol Chem Sci., 2014, 5, 444

10. Pramanik, T.; Maji, P. Int J Pharm Pharm Sci., 2015, 7, 376.

11. Pramanik, T.; Padan, S. K. Int J Pharm Pharm Sci., 2016, 8, 396.

12. Pramanik, T.; Padan, S.K.; Gupta, R. Der Pharmacia Lettre, 2016, 8, 74.

13. Khaskel, A.; Gogoi, P.; Barman, P.; Bandyopadhyay, B. RSC Advances, 2014, 64, 35559.

14. Bose, A. K.; Pednekar, S.; Ganguly, S. N.; Manhas, M. S. Tetrahedron Letter, 2004, 45, 8351.

15. Elnabi, A. H.; Hameed, A. M. A.; Awed, R. R. Sadek, K. U. Green and Sustainable Chemistry, 2013, 3, 141. 